\title{
Efficient Use of Joint Source-Destination Cooperation in the Gaussian Multiple Access Channel
}

\author{
Ahmad Abu Al Haija \\ ECE Department, McGill University, Montreal, QC, Canada \\ Email: ahmad.abualhaija@mail.mcgill.ca
}

\author{
Mai $\mathrm{Vu}$ \\ ECE Department, Tufts University, Medford, MA, USA \\ Email:maivu@ece.tufts.edu
}

\begin{abstract}
We consider the impact of destination cooperation on improving the achievable rate region in a multiple access channel with joint source-destination cooperation (MAC-SDC). Such cooperation may be appealing in systems with a more powerful destination than the sources, such as the uplink of cellular networks. We propose a coding scheme in which each source employs superposition block Markov encoding and partial decode-forward relaying, while the destination employs quantizeforward relaying and backward decoding. The sources partially exchange their messages using not only the direct links between them as in source cooperation, but also the feedback links from the destination, hence they are able to exchange more information at the same power by utilizing the destination as a relay. We analyze the condition on channel and power parameters such that involving destination cooperation is strictly better than just having source cooperation. Results for Gaussian channels show improvement especially when the inter-source link qualities are close to source-destination link qualities. These results provide guideline for practical systems such as cellular networks in determining when it is beneficial for the base station to cooperate with mobile users in the uplink communication.
\end{abstract}

\section{INTRODUCTION}

The multiple access channel (MAC) models practical communication systems such as the uplink communication in cellular networks. Extensive research [1]-[4] shows that cooperation among the two sources increases the achievable region in both full and half-duplex cases. In [1], Willems et al. propose a coding scheme for the MAC with generalized feedback (MAC-GF), also referred to as the MAC with source cooperation (MAC-SC). The proposed scheme in [1] is based on superposition block Markov encoding, partial decodeforward (PDF) relaying and backward decoding. Sendonaris et al. in [2] adapt this coding scheme into cellular networks and show that cooperation can increase the achievable rate region and the cellular coverage as well as decrease the outage probability. After that, Li et al. in [4] propose a hybrid compress-forward (CF) and decode-forward (DF) scheme for this channel where one source employs $\mathrm{CF}$ relaying and the other source employs DF relaying. In [3], we propose a halfduplex scheme which can be applied directly in practical systems such as cellular networks without duplex adaptation.

In cellular networks, the destination (base station) is usually more powerful and has more capabilities than the sources. Hence, it is also of interest to study the impact of destination cooperation on the achievable rates. The destination cooperation has been studied for the MAC where the same feedback signals received by the two sources in [5], [6]. With noiseless direct feedbacks, it is shown in [6] that the capacity of the Gaussian channel is achievable even without inter-user links. Destination cooperation is also used for the interference channel (IC) in [7], [8], where the two destinations cooperate to mitigate the interference and increase the achievable rate region. Destination and relay cooperation are also considered in [9] for the multiple access relay channel (MARC) and in [10], [11] for the relay channel. We show in [11] that destination cooperation in the relay channel can asymptotically achieve the capacity as the destination power approaches infinity.

In this paper, we study the multiple access channel with joint source destination cooperation (MAC-SDC). We propose a coding scheme for this channel and derive its achievable rate region. The transmission is carried over $B$ independent blocks. The destination compresses the received signal in each block and forwards it to the sources but starts decoding only at the end of the last block using backward decoding. Thus, different from [1] where the destination only decodes, here it also produces an input to the channel. The destination employs the quantize-forward technique as in [12], [13] which does not bin the compression index as in the regular $\mathrm{CF}$ technique in [14] and leads to a larger rate region. Each source utilizes the received signals from the other source and the destination to decode part of the message sent by the other. Then, it forwards this message part with its new message to the destination in the next block. The achievable rate region includes as special cases the MAC-SC [1] and the TWRC [12].

We apply the coding scheme into the Gaussian channel and show that joint source destination cooperation improves the rate region compared to just source cooperation especially when the inter-source link qualities are close to each of the source-destination link qualities. Moreover, for symmetric channels, we derive the maximum inter-source link quality at which destination cooperation is useful and can increase the achievable rates significantly. These results are helpful for practical systems such as cellular, sensor and ad hoc networks.

\section{Channel Model}

The MAC-SDC consists of 3 input alphabets $\mathcal{X}_{1}, \mathcal{X}_{2}$ and $\mathcal{X}_{d}, 3$ output alphabets $\mathcal{Y}_{1}, \mathcal{Y}_{2}$, and $\mathcal{Y}_{d}$ and is characterized by the transition probability $p\left(y_{d}, y_{1} . y_{2} \mid x_{1}, x_{2}, x_{d}\right)$. In addition to the 2 direct links from each source to the destination, there are 4 cooperative links: 2 inter-source links and 2 feedback links from the destination to each source. Each source has an encoding function while the destination has, in addition to the decoding function, an encoding function to send feedback 


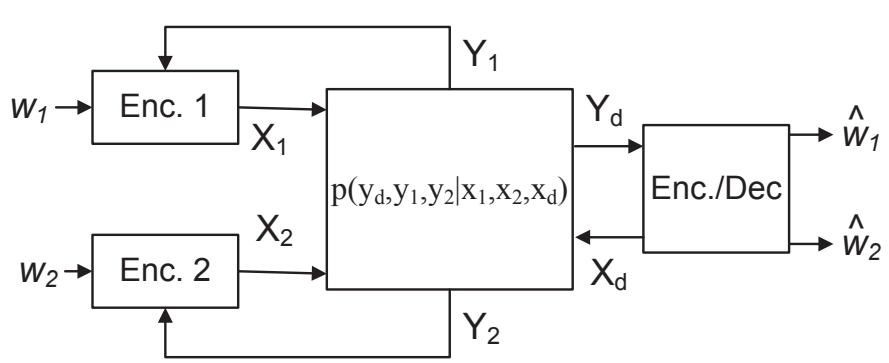

Fig. 1. The channel model for the MAC-SDC.

information to the sources. Figure 1 shows the channel model for the discrete memoryless MAC-SDC. The full definition is in Appendix A.

For the the Gaussian channel with full-duplex operation at each node, the channel model for the MAC-SDC is given as

$$
\begin{aligned}
& Y_{1}=h_{21} X_{2}+h_{01} X_{d}+Z_{1}, \\
& Y_{2}=h_{12} X_{1}+h_{02} X_{d}+Z_{2}, \\
& Y_{d}=h_{10} X_{1}+h_{20} X_{2}+Z_{d}
\end{aligned}
$$

where $\left(h_{12}, h_{21}\right),\left(h_{10}, h_{01}\right)$, and $\left(h_{20}, h_{02}\right)$ are complex link coefficient pairs between the node pairs $\left(S_{1}, S_{2}\right),\left(S_{1}, D\right)$, and $\left(S_{2}, D\right)$, respectively; $Z_{l} \sim \mathcal{C N}(0,1), l \in\{1,2, d\}$ are independent complex AWGN. Each link coefficient is a complex value $h_{i j}=g_{i j} e^{\sqrt{-1} \theta_{i j}}$ where $g_{i j}$ is the real amplitude gain and $\theta_{i j}$ is the phase. We assume that both sources and the destination know all the link amplitude gains and can perfectly compensate the phases.

\section{A CODing SCHEME FOR MAC-SDC}

The proposed coding scheme aims to utilize the destination cooperation to increase the rates at which the sources can exchange their messages. In the MAC-SC, the sources exchange their messages using the direct links between them as in a single-user channel. However, in the MAC-SDC, the sources can exchange their messages using not only the direct links but also the feedback links from destination as in the two-way relay channel (TWRC). By using the destination as a relay, the sources can exchange their messages at higher rates than the MAC-SC.

A scheme for the MAC-SDC is designed such that at each source, the coding is based on rate splitting, superposition block Markov encoding, PDF relaying and sliding window joint decoding over two consecutive blocks. At the destination, the coding is based on QF relaying and backward decoding. Next, we describe the coding scheme in more details with help from Figure 2 and Table I.

The transmission is done in $B$ independent blocks of length $n$. Since the encoding is symmetric at each source, we describe the encoding at $S_{1}$ only. $S_{1}$ splits its message into two parts: a private message $\left(w_{10}\right)$ at rate $\left.R_{10}\right)$ and a public message $\left(w_{11}\right)$ at rate $R_{11}$. In block $i, S_{1}$ knows the message pair $\left(w_{11, i-2}, w_{21, i-2}\right)$ of 2 blocks ago and generates a codeword $U$ for each pair. Then, it superimposes $w_{11, i}$ of the current block on $U$ to generate $V_{1}$. Next, it superimposes $w_{10, i}$ on both $U$ and $V_{1}$ to generate $X_{1}$. Moreover, in this block, $S_{1}$ decodes $w_{21, i-1}$ from its received signals in block $i$ and $i+1$

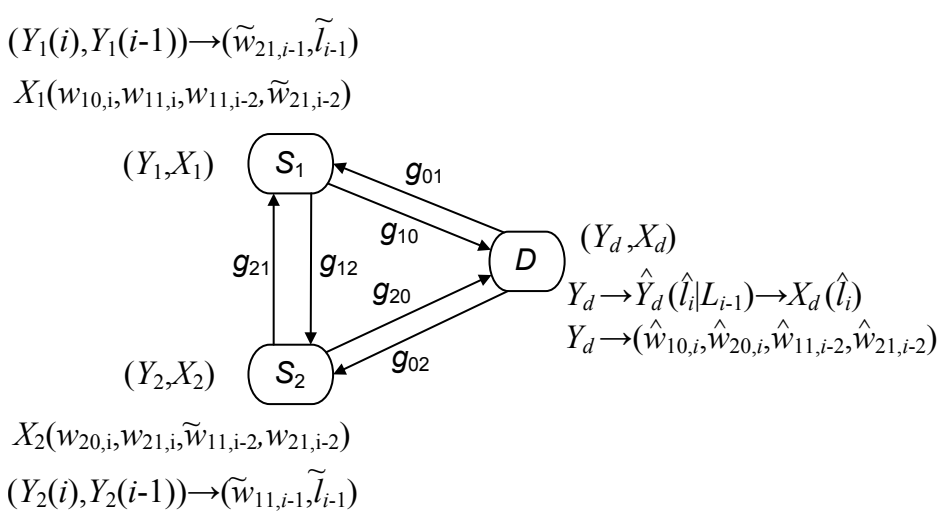

Fig. 2. The coding scheme for the MAC-SDC in block $i$.

using sliding window joint decoding as in [12]. Superposition encoding can be used efficiently here because the destination is interested in decoding all the message parts from both sources.

The destination relays what it receives in each block using QF relaying. However, it only decodes at the end of the last transmitted block using backward decoding. Specifically, in block $i, D$ already knows the quantization index of the previous block. It simply quantizes its received signal in the current block and forwards in the next block by transmitting the codeword $X_{d}$. Appendix A formally describes the encoding and decoding in detail.

\section{A. Achievable Rate Region}

Theorem 1. The achievable rate region of the MAC-SDC is the convex closure of rate-tuples $\left(R_{10}, R_{11}, R_{20}, R_{21}\right)$ satisfying

$$
\begin{aligned}
R_{11} & \left.\leq \min \left\{J_{1}, J_{2}\right)\right\}=I_{1}, \\
R_{21} & \left.\leq \min \left\{J_{3}, J_{4}\right)\right\}=I_{2}, \\
R_{10} & \leq I\left(X_{1} ; Y_{d} \mid V_{1}, U, X_{2}\right)=I_{3}, \\
R_{20} & \leq I\left(X_{2} ; Y_{d} \mid V_{2}, U, X_{1}\right)=I_{4}, \\
R_{10}+R_{20} & \leq I\left(X_{1}, X_{2} ; Y_{d} \mid U, V_{1}, V_{2}\right)=I_{5}, \\
R_{1}+R_{2} & \leq I\left(X_{1}, X_{2} ; Y_{d}\right)=I_{6}
\end{aligned}
$$

subject to

$$
\begin{aligned}
& I\left(\hat{Y}_{d} ; Y_{d} \mid X_{1}, V_{2}, U, X_{d}, Y_{1}\right) \leq I\left(X_{d} ; Y_{1} \mid X_{1}, U\right), \\
& I\left(\hat{Y}_{d} ; Y_{d} \mid V_{1}, X_{2}, U, X_{d}, Y_{2}\right) \leq I\left(X_{d} ; Y_{2} \mid X_{2}, U\right),
\end{aligned}
$$

where

$$
\begin{aligned}
& J_{1}=I\left(V_{1} ; \hat{Y}_{d}, Y_{2} \mid X_{2}, U, X_{d}\right) \\
& J_{2}=I\left(V_{1}, X_{d} ; Y_{2} \mid X_{2}, U\right)-I\left(\hat{Y}_{d} ; Y_{d} \mid V_{1}, X_{2}, U, X_{d}, Y_{2}\right), \\
& J_{3}=I\left(V_{2} ; \hat{Y}_{d}, Y_{1} \mid X_{1}, U, X_{d}\right) \\
& J_{4}=I\left(V_{2}, X_{d} ; Y_{1} \mid X_{1}, U\right)-I\left(\hat{Y}_{d} ; Y_{d} \mid X_{1}, V_{2}, U, X_{d}, Y_{1}\right),
\end{aligned}
$$

for some joint distribution that factors as

$$
\begin{aligned}
P^{\dagger}= & p(u) p\left(v_{1} \mid u\right) p\left(v_{2} \mid u\right) p\left(x_{d}\right) p\left(x_{1} \mid v_{1}, u\right) p\left(x_{2} \mid v_{2}, u\right) \\
& p\left(y_{1}, y_{2}, y_{d} \mid x_{1}, x_{2}, x_{d}\right) p\left(\hat{y}_{d} \mid x_{d}, y_{d}\right) .
\end{aligned}
$$

Proof: See Appendix A. 


\begin{tabular}{|c|c|c|c|}
\hline Block & $i-1$ & $i$ & $i+1$ \\
\hline$S_{1}$ & $x_{1}^{n}\left(w_{10}^{i-1}, w_{11}^{i-1}, w_{11}^{i-3}, \tilde{w}_{21}^{i-3}\right)$ & $x_{1}^{n}\left(w_{10}^{i}, w_{11}^{i}, w_{11}^{i-2}, \tilde{w}_{21}^{i-2}\right)$ & $x_{1}^{n}\left(w_{10}^{i+1}, w_{11}^{i+1}, w_{11}^{i-1}, \tilde{w}_{21}^{i-1}\right)$ \\
\hline$S_{2}$ & $x_{2}^{n}\left(w_{20}^{i-1}, w_{21}^{i-1}, \tilde{w}_{11}^{i-3}, w_{21}^{i-3}\right)$ & $x_{2}^{n}\left(w_{20}^{i}, w_{21}^{i}, \tilde{w}_{11}^{i-2}, w_{21}^{i-2}\right)$ & $x_{2}^{n}\left(w_{20}^{i+1}, w_{21}^{i+1}, \tilde{w}_{11}^{i-1}, w_{21}^{i-1}\right)$ \\
\hline$D$ & $\hat{y}_{d}^{n}\left(\hat{l}_{i-1} \mid l_{i-2}\right), x_{d}^{n}\left(\hat{l}_{i-2}\right)$ & $\hat{y}_{d}^{n}\left(\bar{l}_{i} \mid l_{i-1}\right), x_{d}^{n}\left(\hat{l}_{i-1}\right)$ & $\hat{y}_{d}^{n}\left(\hat{l}_{i+1} \mid \hat{l}_{i}\right), x_{d}^{n}\left(\hat{l}_{i}\right)$ \\
\hline$Y_{1}$ & $\left(\tilde{w}_{21}^{i-2}, \tilde{l}_{i-2}\right)$ & $\left(\tilde{w}_{21}^{i-1}, \tilde{l}_{i-1}\right)$ & $\left(\tilde{w}_{21}^{i}, \tilde{l}_{i}\right)$ \\
\hline$Y_{2}$ & $\left(\tilde{w}_{11}^{i-2}, \tilde{l}_{i-2}\right)$ & $\left(\tilde{w}_{11}^{i-1}, \hat{l}_{i-1}\right)$ & $\left(\tilde{w}_{11}^{i}, \tilde{l}_{i}\right)$ \\
\hline$Y_{d}$ & $\leftarrow\left(\hat{w}_{10}^{i-1}, \hat{w}_{20}^{i-1}, \hat{w}_{11}^{-3}, \hat{w}_{21}^{i-3}\right)$ & $\leftarrow\left(\hat{w}_{10}^{i}, \hat{w}_{20}^{i}, \hat{w}_{11}^{i-2}, \hat{w}_{21}^{i-2}\right)$ & $\leftarrow\left(\hat{w}_{10}^{i+1}, \hat{w}_{20}^{i+1}, \hat{w}_{11}^{i-1}, \hat{w}_{21}^{i-1}\right)$ \\
\hline
\end{tabular}

Table I: The encoding and decoding techniques of the proposed scheme for the MAC-SDC.

\section{B. Discussion}

Remark 1. The proposed scheme includes as special cases the achievable rate regions of

- The MAC-SC [1]: by setting $X_{d}=\hat{Y}_{d}=\emptyset$ and $R_{d}=0$.

- The TWRC [12]: by setting $X_{1}=V_{1}, X_{2}=V_{2}, R_{10}=$ $R_{20}=0$, and by removing the decoding at $D$.

Remark 2. For the MAC-GF [1], the transmitted codeword in block $i$ depends only on the codeword transmitted in block $(i-1)$. However, in the proposed scheme, the transmitted codeword at each source depends on the codewords of both previous 2 blocks. This $2^{\text {nd }}$-order block Markov encoding is used because each source decodes the public message part sent 2 blocks ago (block $i-2$ ) by employing joint decoding of the received signals over the last 2 consecutive blocks $(i-2, i-1)$.

Remark 3. In [1], the decoding at each source is the same as in a single-user channel. However, in the proposed scheme, the decoding at each source is similar to the CF lower bound of the relay channel [14], which leads to larger rate than single-user direct transmission.

Remark 4. In the proposed scheme, we use QF relaying at the destination which leads to higher rates than CF relaying. In $\mathrm{CF}$ relaying, the quantization index is further binned to fit the weaker between the two links from the destination to the two sources and hence limits the achievable rates. In QF relaying, the quantization index needs not to be binned nor decoded separately at each source, but can be decoded jointly with messages, hence places no constraint on the rates.

Remark 5. Another scheme for this channel model can be obtained as a special case of the noisy network coding (NNC) scheme in [13]. This scheme employs QF relaying at all nodes, message repetition in all transmission blocks and simultaneous joint decoding over all transmission blocks at the destination. By applying Theorem 1 in [13] into the MAC-SDC, we obtain an achievable rate region as a convex closure of the rate pairs $\left(R_{1}, R_{2}\right)$ satisfying

$$
\begin{aligned}
& R_{1} \leq I\left(X_{1} ; \hat{Y}_{2}, Y_{d} \mid X_{2}, X_{d}\right)-I\left(Y_{1} ; \hat{Y}_{1} \mid X_{1}, X_{2}, X_{d}, \hat{Y}_{2}, \hat{Y}_{d}, Y_{d}\right) \\
& R_{2} \leq I\left(X_{2} ; \hat{Y}_{1}, Y_{d} \mid X_{1}, X_{d}\right)-I\left(Y_{2} ; \hat{Y}_{2} \mid X_{1}, X_{2}, X_{d}, \hat{Y}_{2}, \hat{Y}_{d}, Y_{d}\right) \\
& R_{1}+R_{2} \leq I\left(X_{1}, X_{2} ; Y_{d} \mid X_{d}\right) \\
& \quad-I\left(Y_{1}, Y_{2} ; \hat{Y}_{1}, \hat{Y}_{2} \mid X_{1}, X_{2}, X_{d}, \hat{Y}_{d}, Y_{d}\right)
\end{aligned}
$$

for some $p\left(x_{1}\right) p\left(\hat{y}_{1} \mid x_{1}, y_{1}\right) p\left(x_{2}\right) p\left(\hat{y}_{2} \mid x_{2}, y_{2}\right) p\left(x_{d}\right) p\left(\hat{y}_{d} \mid x_{d}, y_{d}\right)$ $p\left(y_{1}, y_{2}, y_{d} \mid x_{1}, x_{2}, x_{d}\right)$. Depending on the link strength, the individual rates in this NNC region can be bigger or smaller than the individual rates in Theorem 1. However, the NNC sum rate constraint in (6) can be at most as the classical MAC while our scheme achieves a higher sum rate. Our proposed scheme includes the MAC-GF scheme [1] as a special case, which has a higher sum rate than the classical MAC [2].

\section{Coding For Gaussian Channels}

\section{A. Signaling}

The Gaussian MAC-SDC model is given in (1). In the proposed scheme, $S_{1}$ and $S_{2}$ construct their transmit signals $X_{1}$ and $X_{2}$ in block $i$ as follows.

$X_{1}=\sqrt{\rho_{10}} T_{1}\left(w_{10, i}\right)+\sqrt{\rho_{11}} V_{1}\left(w_{11, i}\right)+\sqrt{\rho_{1}} U\left(w_{11, i-2}, w_{21, i-2}\right)$, $X_{2}=\sqrt{\rho_{20}} T_{2}\left(w_{20, i}\right)+\sqrt{\rho_{21}} V_{2}\left(w_{21, i}\right)+\sqrt{\rho_{2}} U\left(w_{11, i-2}, w_{21, i-2}\right)$,

where $T_{1}, T_{2}, V_{1}, V_{2}$ and $U$ are i.i.d random variables $\sim$ $\mathcal{N}(0,1)$. The power constraints at the two sources are

$$
\rho_{10}+\rho_{11}+\rho_{1}=P_{1}, \quad \rho_{20}+\rho_{21}+\rho_{2}=P_{2} .
$$

The destination constructs the quantized received signal $\hat{Y}_{d}$ as $\hat{Y}_{d}=Y_{d}+\hat{Z}_{d}$ and the transmit signal as $X_{d} \sim \mathcal{N}\left(0, P_{d}\right)$. For $\mathrm{QF}$ techniques, the optimal $\hat{Z}_{d}$ may not be Gaussian; however, here we consider $\hat{Z}_{d} \sim \mathcal{N}\left(0, \sigma^{2}\right)$.

\section{B. Achievable Rate Region}

Theorem 2. The achievable rate region for the Gaussian $M A C-S D C$ can be expressed as in (2) with

$I_{1}=\min \left\{J_{1}, J_{2}\right\}, \quad I_{2}=\min \left\{J_{3}, J_{4}\right\}$,

$I_{3}=\mathcal{C}\left(g_{10}^{2} \rho_{10}\right), \quad I_{4}=\mathcal{C}\left(g_{20}^{2} \rho_{20}\right), \quad I_{5}=\mathcal{C}\left(g_{10}^{2} \rho_{10}+g_{20}^{2} \rho_{20}\right)$,

$I_{6}=\mathcal{C}\left(g_{10}^{2} P_{1}+g_{20}^{2} P_{2}+2 g_{10} g_{20} \sqrt{\rho_{1} \rho_{2}}\right)$

where $\mathcal{C}(x)=\log (1+x)$ and

$$
\begin{aligned}
& J_{1}=\mathcal{C}\left(\frac{g_{10}^{2} \rho_{11}+\left(1+\sigma^{2}\right) g_{12}^{2} \rho_{11}}{\left(1+\sigma^{2}\right)\left(1+g_{12}^{2} \rho_{10}\right)+g_{10}^{2} \rho_{10}}\right), \\
& J_{2}=\mathcal{C}\left(\frac{g_{12}^{2} \rho_{11}+g_{02}^{2} P_{d}}{1+g_{12}^{2} \rho_{10}}\right)-\mathcal{C}\left(\frac{1+\left(g_{12}^{2}+g_{10}^{2}\right) \rho_{10}}{\sigma^{2}\left(1+g_{12}^{2} \rho_{10}\right)}\right), \\
& J_{3}=\mathcal{C}\left(\frac{g_{20}^{2} \rho_{21}+\left(1+\sigma^{2}\right) g_{21}^{2} \rho_{21}}{\left(1+\sigma^{2}\right)\left(1+g_{21}^{2} \rho_{20}\right)+g_{20}^{2} \rho_{20}}\right), \\
& J_{4}=\mathcal{C}\left(\frac{g_{21}^{2} \rho_{21}+g_{01}^{2} P_{d}}{1+g_{21}^{2} \rho_{20}}\right)-\mathcal{C}\left(\frac{1+\left(g_{21}^{2}+g_{20}^{2}\right) \rho_{20}}{\sigma^{2}\left(1+g_{21}^{2} \rho_{20}\right)}\right),
\end{aligned}
$$

These constraints are subject to the conditions in (3) which are expressed in Gaussian channels as

$$
\sigma_{c} \geq \max \left\{\sigma_{1}, \sigma_{2}\right\} \text { where }
$$




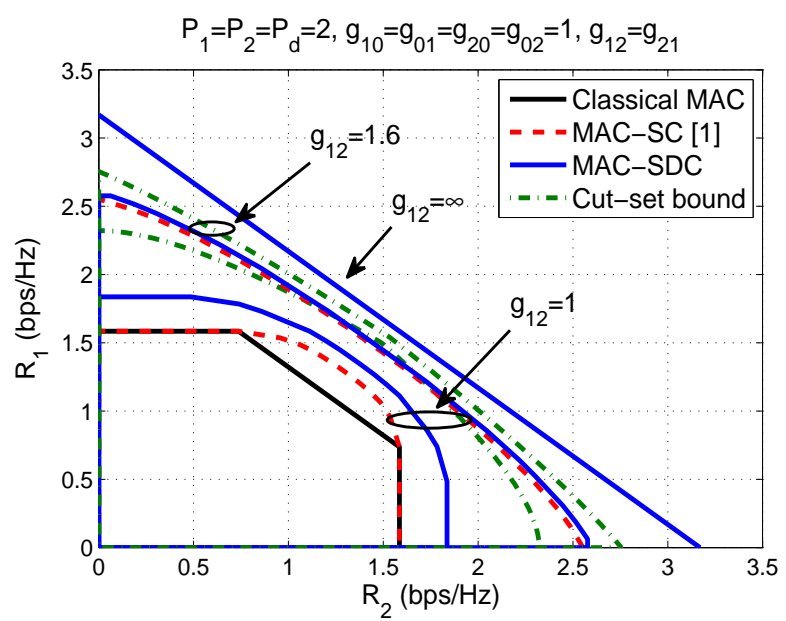

Fig. 3. Comparison of the proposed scheme, MAC-SC [1], classical MAC and the cut-set bound.

$$
\begin{aligned}
\sigma_{1} & =\frac{\left(1+g_{12}^{2}\left(\rho_{10}+\rho_{12}\right)\right)\left(1+\left(g_{12}^{2}+g_{10}^{2}\right) \rho_{10}\right)}{\left(1+g_{12}^{2} \rho_{10}\right) g_{02}^{2} P_{d}}, \\
\sigma_{2} & =\frac{\left(1+g_{21}^{2}\left(\rho_{20}+\rho_{21}\right)\right)\left(1+\left(g_{21}^{2}+g_{20}^{2}\right) \rho_{20}\right)}{\left(1+g_{21}^{2} \rho_{20}\right) g_{01}^{2} P_{d}}
\end{aligned}
$$

Proof: By direct application of Theorem 1 into the Gaussian channel with signaling in Section IV-A.

Remark 6. As $P_{d} \rightarrow \infty$, the individual rates of this scheme achieve the capacity by reaching the cut-set bound. For the individual rate, when $P_{d} \rightarrow \infty$, the destination virtually joins a source in one entity because of the infinite feedback link. Thus, the cut-set bound for the relay channel is asymptotically achieved. See [11] for more details of the proof.

Figure 3 compares between the achievable rate region of the proposed scheme for the MAC-SDC, and those of the MAC-SC [1], classical MAC and the cut-set bound for MAC-SDC. Although Figure 3 is for symmetric channels, similar results can be obtained for asymmetric channels by applying Theorem 2. Results show that joint source-destination cooperation improves the achievable region compared with source cooperation alone. We note that the improvement diminishes as the inter-source link qualities increase. This is intuitive because when the inter-source links are very strong, the sources can exchange their messages without help from the destination. Therefore, for practical systems, joint sourcedestination cooperation is preferable when the inter-source link qualities are close to each source-destination link quality in which source cooperation alone brings little improvement.

\section{Optimal Use of Destination Cooperation}

From Figure 3, we see that destination cooperation is most beneficial when the inter-user links are comparable with the source-destination links. In this section, we find the threshold on the inter-source link qualities $\left(g_{12}\right.$, and $\left.g_{21}\right)$ for the rate region of the MAC-SDC to be the same as of the MAC-SC.

The rate region for the Gaussian MAC-SC [2] are as in (8) but without the condition in (10) and with

$$
I_{1}=\mathcal{C}\left(\frac{g_{12}^{2} \rho_{11}}{1+g_{12}^{2} \rho_{10}}\right), \quad I_{2}=\mathcal{C}\left(\frac{g_{21}^{2} \rho_{21}}{1+g_{21}^{2} \rho_{20}}\right) .
$$

To compare between the two regions, we need to consider condition (10) in Theorem 8 and compare $I_{1}$ and $I_{2}$ in the two regions. Lets first find the optimal $\sigma$ that maximizes $I_{1}$ in (8). Since $J_{2}$ is an increasing function of $\sigma$ and $J_{1}$ is a decreasing function, the optimal $\sigma$ is obtained from the intersection between $J_{1}$ and $J_{2}$ as

$$
\sigma_{12}^{2}=\frac{1+\left(g_{10}^{2}+g_{12}^{2}\right)\left(\rho_{10}+\rho_{11}\right)}{g_{02}^{2} P_{d}}
$$

Similarly, $I_{2}$ is maximized with the $\sigma_{34}^{2}$ resulting from the intersection between $J_{3}$ and $J_{4}$.

By substituting $\sigma^{2}$ in (12) into $J_{1}$ or $J_{2}$ in (9), we obtain $I_{1}$ as

$$
I_{1}=\mathcal{C}\left(\frac{1}{1+g_{12}^{2} \rho_{10}}\left[g_{12}^{2} \rho_{11}+\frac{\rho_{11}}{\rho_{10}}(1-(1 / \beta))\right]\right)
$$

where

$$
\begin{aligned}
& \beta=1+\mu, \\
& \mu=\frac{g_{10}^{2} g_{02}^{2} \rho_{10} P_{d}}{\left(1+g_{12}^{2} \rho_{10}\right)\left(1+\left(g_{12}^{2}+g_{10}^{2}\right)\left(\rho_{10}+\rho_{11}\right)+g_{02}^{2} P_{d}\right)}
\end{aligned}
$$

By comparing between $I_{1}$ in (11) and (13), they are almost the same if $\mu$ in (14) is very small, i.e. $\mu \leq \varepsilon$, where $\varepsilon$ is a very small number. This condition is equivalent to

$$
\begin{aligned}
g_{12 t}^{2} & \geq \frac{-a_{2}+\sqrt{a_{2}^{2}-4 a_{1} a_{3}}}{2 a_{1}}, \text { with } & & \\
a_{1} & =\varepsilon \rho_{10}\left(\rho_{10}+\rho_{11}\right), & a_{2} & =\varepsilon\left(\rho_{10}\left(2+a_{4}\right)+\rho_{11}\right), \\
a_{3} & =\varepsilon\left(1+a_{4}\right)-g_{10}^{2} g_{02}^{2} \rho_{10} P_{d}, & a_{4} & =g_{10}^{2}\left(\rho_{10}+\rho_{11}\right)+g_{02}^{2} P_{d} .
\end{aligned}
$$

Now, from (10) and (12), it is easy to show that $\sigma_{12}>\sigma_{1}$ and $\sigma_{34}>\sigma_{2}$. However, $\sigma_{12}$ is different from $\sigma_{34}$ and is not comparable with $\sigma_{2}$. Similarly, $\sigma_{34}$ is not comparable with $\sigma_{1}$. Therefore, it is not easy to determine the optimal $\sigma$ and hence the condition on the inter-source links that leads to the same rate region for the MAC-SDC as the MAC-SC. To overcome this difficulty, we limit our analysis into the symmetric channel where $g_{12}=g_{21}, g_{10}=g_{20}=g_{01}=g_{02}$ and $P_{1}=P_{2}$. Moreover, we only consider the maximum individual and sum rate points in the rate region and not the entire rate region.

- The maximum sum rate is obtained with the same power allocations from both users $\left(\rho_{10}=\rho_{20}, \rho_{11}=\rho_{21}\right.$, and $\left.\rho_{1}=\rho_{2}\right)$. Therefore, $\sigma_{12}=\sigma_{34}, \sigma_{1}=\sigma_{2}$, and $\sigma_{12}>\sigma_{1}$.

- For the individual rate, lets consider $R_{1}$ which is maximized when $R_{2}=0$, i.e., $S_{2}$ does not have any message to send and just relays the message of $S_{1}$. Then, the optimal power allocations for $S_{2}$ are

$$
\rho_{20}=\rho_{21}=0 \text {, and } \rho_{2}=P_{2} \text {. }
$$

With these power allocations at $S_{2}$, then $I_{2}=I_{4}=0$ and $\sigma_{2}=\left(g_{01}^{2} P_{d}\right)^{-1}$. Thus, $\sigma_{12}>\max \left(\sigma_{1}, \sigma_{2}\right)$.

Hence, we obtain the following corollary:

Corollary 1. In the symmetric MAC-SDC, joint sourcedestination cooperation leads to a significant improvement over source cooperation for the individual and the sum rates if the inter-source link quality $\left(g_{12}\right)$ is less than $g_{12 t}$ in (15). 


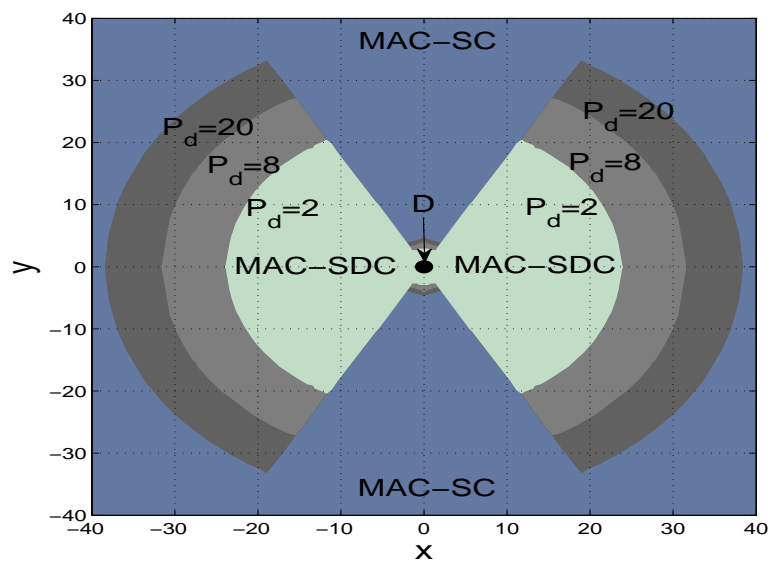

Fig. 4. Regions for the efficient use of MAC-SDC for maximizing the individual and sum rates with $P_{1}=P_{2}=2$ and different $P_{d}$.

However, if $g_{12}>g_{12 t}$, joint source-destination cooperation adds little improvement over source cooperation alone.

In Figure 4, we consider a $2 D$ network with fixed destination location at $(0,0)$ while letting $S_{1}$ move on the plane, provided that $S_{2}$ is on the opposite $x$ location, i.e., if $S_{1}$ lies at $(x, y)$, then $S_{2}$ lies at $(-x, y)$. We apply the pathloss model in which the channel gain $g_{i j}$ depends only on the distance as $g_{i j}=d_{i j}^{-\gamma / 2}$, where $\gamma=2.4$. This network model preserves the symmetry of the channel. For the inter-source link threshold $\left(g_{12 t}\right)$ in $(15)$, we set $\varepsilon=10^{-6}$. In order to find $g_{12 t}$ and compare it with $g_{12}$, we need to calculate it at the optimal power allocations for the individual or the sum rate which can be obtained numerically.

Results show geometrical regions where the MAC-SDC scheme is useful since it can lead to a significant improvement over MAC-SC. Results also show regions where the MAC-SC is preferred because it is simpler and leads to almost the same region as the MAC-SDC. The regions where the MAC-SDC is preferred is the same for the individual and sum rate, which is expected considering the case of $g_{12}=1.6$ in Figure 3 . Furthermore, the MAC-SDC geometrical region increases as the destination power $\left(P_{d}\right)$ increases. Since there is a preferred geometrical region for the MAC-SDC even when $P_{d}=P_{1}$, joint source-destination cooperation can also be used in sensor and ad hoc networks, where all nodes usually have comparable transmit powers. Increasing $P_{d}$ enlarges the geometrical region and increases the achievable rates as well.

Figures 5 and 6 illustrate the gaps in the individual and the sum rates between the MAC-SDC and MAC-SC schemes. Results show that the gap increases as $P_{d}$ increases. Moreover, the gap increases with $g_{12}$ as $g_{12}<g_{10}$ and starts decreasing as $g_{12}>g_{10}$. For the individual rate, the maximum gap always occurs at $g_{12}=1$ because the MAC-SC scheme has no effect on the individual rate as $g_{12}<g_{10}$. However, for the sum rate, the maximum gap does not occur at $g_{12}=1$ but varies with $P_{d}$ because the MAC-SDC and MAC-SC improve the sum rate even when $g_{12}<g_{10}$. The maximum individual and sum rate gaps at $P_{d} \rightarrow \infty$ are $0.75 \mathrm{bps} / \mathrm{Hz}$ and $0.42 \mathrm{bps} / \mathrm{Hz}$, respectively.

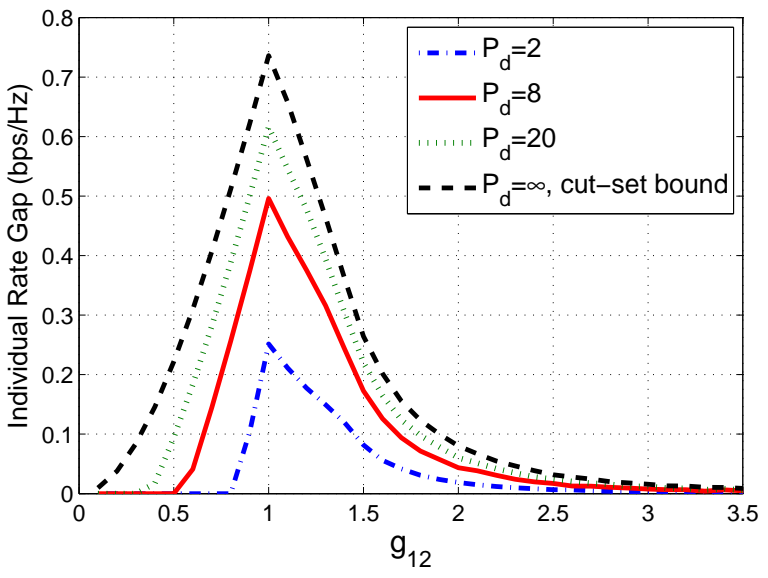

Fig. 5. Gaps in the individual rates between the MAC-SDC and MAC-SC versus $g_{12}$ for symmetric channels with $P_{1}=P_{2}=2, g_{10}=1$ and different $P_{d}$.

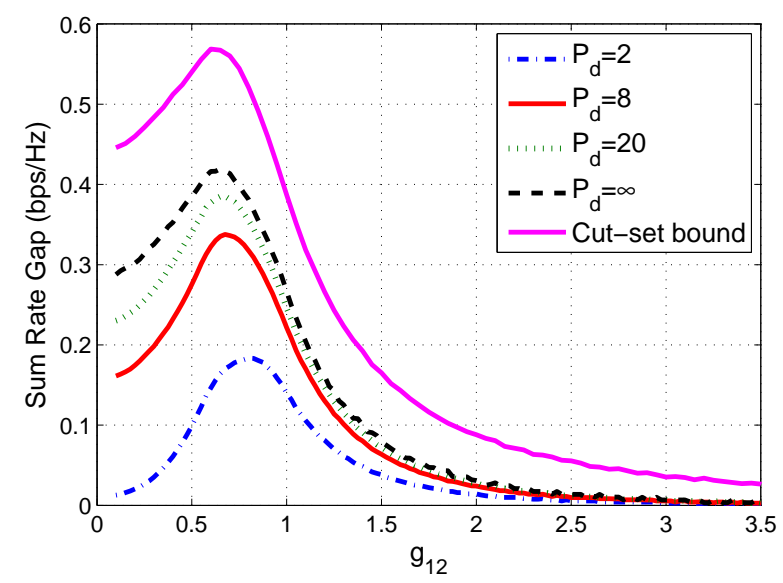

Fig. 6. Gaps in the sum rates between the MAC-SDC and MAC-SC versus $g_{12}$ for symmetric channels with $P_{1}=P_{2}=2, g_{10}=1$ and different $P_{d}$.

\section{CONCLUSiON}

We have proposed a new coding scheme for the MACSDC based on message splitting, superposition block Markov encoding, and PDF relaying at the each source, and QF relaying and backward decoding at the destination. We analyze the achievable rate region in detail and show that it includes as special cases the achievable regions of the MAC-SC and the TWRC obtained with QF technique. We also present numerical results that show improvement on the rate region for the MACSDC compared with the MAC-SC. We derive a condition for the inter-source link quality at which the MAC-SDC can lead to a significant improvement over the MAC-SC. Moreover, we provide numerical results that determine the rate gaps between the MAC-SDC and MAC-SC, and the geometrical regions where the MAC-SDC scheme is preferred. Studying the optimal power allocation and the impact of fading channels on the achievable rate regions, the geometrical regions and the rate gaps will be of interest for future works.

\section{APPENDIX A PROOF OF THEOREM 1}

In order to prove Theorem 1, we describe here the formal coding scheme with help from Figure 2 and Table I. A 
$\left(\left\lceil 2^{n R_{1}}\right\rceil,\left\lceil 2^{n R_{2}}\right\rceil, n\right)$ code for the MAC-SDC channel consists of two independent message sets $W_{1}=\left\{1, \ldots,\left\lceil 2^{n R_{1}}\right\rceil\right\}$, and $W_{2}=\left\{1, \ldots,\left\lceil 2^{n R_{2}}\right\rceil\right\}$, three encoding functions $\left(f_{1 i}, f_{2 i}, f_{3 i} i=1, \ldots, n\right)$, and one decoding function $\left(g_{1}\right)$ defined as $f_{1 i}: W_{1} \times \mathcal{Y}_{1}^{i-1} \rightarrow \mathcal{X}_{1}, f_{2 i}: W_{2} \times \mathcal{Y}_{2}^{i-1} \rightarrow \mathcal{X}_{2}$, $f_{3 i}: \mathcal{Y}_{d}^{i-1} \rightarrow \mathcal{X}_{d}$, and $g_{1}: \mathcal{Y}_{d}^{n} \rightarrow \hat{W}_{1} \times \hat{W}_{2}$, where $i=1, \ldots, n$. Definitions for the average error probability, achievability and capacity follow the standard ones in [14].

We consider $B$ independent transmission blocks each of length $n$. Two sequences of $B-2$ messages $w_{1, i}$ and $w_{2, i}$ for $i \in[1: B-2]$, are to be sent in $n B$ transmissions. No new information is sent in the last two blocks $(B-1$ and $B)$. This reduces the achievable rates in (2) by a factor of $2 / B$; however, as $B \rightarrow \infty$, this factor becomes negligible.

\section{A. Codebook generation}

The codebook generation of the proposed coding scheme in block $i$ can be explained as follows. After fixing $P^{\dagger}$ as in (5),

- Generate $\quad 2^{n\left(R_{11}+R_{21}\right)} \quad$ i.i.d $\quad$ sequences $u^{n}\left(w_{11, i-2}, w_{21, i-2}\right) \sim \prod_{k=1}^{n} p\left(u_{k}\right)$

- For each $u^{n}\left(w_{11, i-2}, w_{21, i-2}\right)$, generate $2^{n R_{11}}$ i.i.d sequences $v_{1}^{n}\left(w_{11, i}, w_{11, i-2}, w_{21, i-2}\right) \sim \prod_{k=1}^{n} p\left(v_{1 k} \mid u_{k}\right)$,

- For each pair $\left(u^{n}\left(w_{11, i-2}, w_{21, i-2}\right)\right.$, $\left.v_{1}^{n}\left(w_{11, i}, w_{11, i-2}, w_{21, i-2}\right)\right), \quad$ generate $2^{n R_{10}}$ i.i.d sequences $\quad x_{1}^{n}\left(w_{10, i}, w_{11, i}, w_{11, i-2}, w_{21, i-2}\right) \sim$ $\prod_{k=1}^{n} p\left(x_{1 k} \mid v_{1 k}, u_{k}\right)$.

- The codewords $v_{2}^{n}$ and $x_{2}^{n}$ are generated in similar way to $v_{1}^{n}$ and $x_{1}^{n}$, respectively.

- Generate $2^{n R_{d}}$ i.i.d sequences $x_{d}^{n}\left(l_{i-1}\right) \sim \prod_{k=1}^{n} p\left(x_{d k}\right)$.

- For each $x_{d}^{n}\left(l_{i-1}\right)$, generate $2^{n R_{d}}$ i.i.d sequences $\hat{y}_{d}^{n}\left(l_{i} \mid l_{i-1}\right) \sim \prod_{k=1}^{n} p\left(\hat{y}_{k} \mid x_{d, k}\right)$.

\section{B. Encoding}

Let $\left(w_{10, i}, w_{11, i}, w_{20, i}, w_{21, i}\right)$ be the new messages to be sent in block $i . S_{1}$ has an estimate $\left(\hat{w}_{21, i-2}\right)$ of $w_{21, i-2}$. Similarly holds for $S_{2}$ and $w_{11, i-2}$. Then, $S_{1}$ sends the message vector $\left(w_{10, i}, w_{11, i}, w_{11, i-2}, \hat{w}_{21, i-2}\right)$ by transmitting $x_{1}^{n}\left(w_{10, i}, w_{11, i}, w_{11, i-2}, \hat{w}_{21, i-2}\right)$. Similarly, $S_{2}$ transmits $x_{2}^{n}\left(w_{20, i}, w_{21, i}, \hat{w}_{11, i-2}, w_{21, i-2}\right)$. The destination has estimated $\hat{l}_{i-1}$ in block $i-1$; hence, in block $i$, it sends $x_{d}^{n}\left(\hat{l}_{i-1}\right)$.

\section{Decoding}

Without loss of generality, assume that all transmitted messages and the compression indices are equal to 1 . Then, the decoding can be described as follows.

1) At Each Source: The decoding at each source is quite similar to that proposed in [12] for the TWRC. At the end of block $i, S_{1}$ already knows $l_{i-1}=L_{i-1}, w_{21, i-2}=1$ and $w_{21, i-1}=1$ from the decoding in blocks $i-1$ and $i$. Then, $S_{1}$ employs joint decoding over blocks $i$ and $i+1$ to find a unique pair $\left(\hat{w}_{21, i}, \hat{l}_{i}\right)$ such that

$$
\begin{array}{r}
\left(v_{2}^{n}\left(\hat{w}_{21, i}, 1,1\right), x_{d}^{n}\left(L_{i-1}\right), \hat{y}_{d}^{n}\left(\hat{l}_{i} \mid L_{i-1}\right), x_{1}^{n}, u^{n}, v_{1}^{n}, y_{1}^{n}(i)\right) \in A_{\epsilon}^{n} \\
\text { and }\left(x_{d}^{n}\left(\hat{l}_{i}\right), x_{1}^{n}, v_{1}^{n}, u^{n}, y_{1}^{n}(i+1)\right) \in A_{\epsilon}^{n}
\end{array}
$$

The error analysis for this decoding rule is given in [12] and it leads to the following rate constraints:

$$
R_{d} \leq I\left(X_{d} ; Y_{1} \mid X_{1}, U\right)+I\left(\hat{Y}_{d} ; X_{1}, V_{2}, U, Y_{1} \mid X_{d}\right),
$$

$$
\begin{aligned}
R_{21} & \leq I\left(V_{2} ; \hat{Y}_{d}, Y_{1} \mid X_{1}, U, X_{d}\right), \\
R_{21}+R_{d} & \leq I\left(V_{2}, X_{d} ; Y_{1} \mid X_{1}, U\right)+I\left(\hat{Y}_{d} ; X_{1}, V_{2}, U, Y_{1} \mid X_{d}\right)
\end{aligned}
$$

Similar decoding rule holds at $S_{2}$.

2) At the Destination: $D$ employs two steps of decoding. First, during the transmission process, $D$ quantizes the received signal from both sources. That is, in block $i$, the destination already knows $l_{i-1}=L_{i-1}$, it finds an index $l_{i}$ such that

$$
\left(\hat{y}_{d}^{n}\left(l_{i} \mid L_{i-1}\right), x_{d}^{n}\left(L_{i-1}\right), y_{d}^{n}(i)\right) \in A_{\epsilon}^{n}
$$

By the covering lemma, such $l_{i}$ exists if $R_{d}>I\left(\hat{Y}_{d} ; Y_{d} \mid X_{d}\right)$. By combining this constraint with (17), we obtain $I_{2}$ and the $2^{\text {nd }}$ inequality in (2) and (3), respectively. Similarly, from the decoding at $S_{2}$, we obtain $I_{1}$ and the $1^{\text {st }}$ inequality in (2) and (3), respectively.

Then, at the end of the last transmission block, the destination employs backward decoding to decode all transmitted messages. In block $i$, the destination already knows $w_{11, i}$ and $w_{21, i}$ from the decoding in block $i+2$. Hence, it declares that the message vector $\left(\hat{w}_{10, i}, \hat{w}_{20, i}, \hat{w}_{11, i-2}, \hat{w}_{21, i-2}\right)$ is sent if it is the unique message vector such that

$$
\begin{gathered}
\left(u^{n}\left(\hat{w}_{11, i-2}, \hat{w}_{21, i-2}\right), v_{1}^{n}\left(1, \hat{w}_{11, i-2}, \hat{w}_{21, i-2}\right),\right. \\
v_{2}^{n}\left(1, \hat{w}_{11, i-2}, \hat{w}_{21, i-2}\right), x_{1}^{n}\left(\hat{w}_{10, i}, 1, \hat{w}_{11, i-2}, \hat{w}_{21, i-2}\right), \\
\left.x_{2}^{n}\left(\hat{w}_{20, i}, 1, \hat{w}_{11, i-2}, \hat{w}_{21, i-2}\right), y_{d}^{n}(i)\right) \in A_{\epsilon}^{n}
\end{gathered}
$$

This decoding rule leads to the rate constraints $I_{3}-I_{6}$ in (2).

\section{REFERENCES}

[1] F. M. J. Willems, E. C. van der Meulen, and J. P. M. Schalkwijk, "Achievable rate region for the multiple-access channel with generalized feedback," in Proc. Annu. Allerton Conf. on Comm., Control and Computing, 1983, pp. 284-292.

[2] A. Sendonaris, E. Erkip, and B. Aazhang, "User cooperation diversity Part I," IEEE Trans. Comm., vol. 51, no. 11, pp. 1927-1938, Nov. 2003.

[3] A. Abu Al Haija and M. Vu, "A half-duplex cooperative scheme with partial decode-forward relaying," in IEEE ISIT, Aug. 2011.

[4] Q. Li, K. Li, and K. Teh, "An achievable rate region of cooperative multiple-access channels with hybrid CF and DF cooperation," IEEE Comm. Letters, vol. 13, no. 8, pp. 591 -593, Aug. 2009.

[5] T. Cover and C. Leung, "An achievable rate region for the multipleaccess channel with feedback," IEEE Trans. Info. Theory, vol. 27, no. 3, pp. $292-298$, May 1981.

[6] L. Ozarow, "The capacity of the white Gaussian multiple access channel with feedback," IEEE Trans. Info. Theory, vol. 30, no. 4, pp. 292 - 298, July 1984.

[7] V. Prabhakaran and P. Viswanath, "Interference channels with destination cooperation," IEEE Trans. Info. Theory, vol. 57, no. 1, pp. 187 -209 , Jan. 2011.

[8] I.-H. Wang and D. Tse, "Interference mitigation through limited receiver cooperation," IEEE Trans. Info. Theory, vol. 57, no. 5, pp. 2913 -2940, May 2011.

[9] J. Wang, Y. Liu, Y. Tan, and B. Zhu, "Joint network-channel design with destination-relay feedback in multiple-access relay channel," in IEEE Internet Technology and Applications, Aug. 2010, pp. 1 -4.

[10] J. Jiang and Y. Xin, "On the achievable rates for the relay channels with generalized feedback," in IEEE CISS 2008, Mar. 2008, pp. 173 -178.

[11] A. Abu Al Haija and M. Vu, "An asymptotically capacity-achieving scheme for the Gaussian relay channel with relay-destination cooperation," in IEEE CISS, Mar. 2013.

[12] P. Zhong, A. Abu Al Haija, and M. Vu, "On compress-forward without Wyner-Ziv binning for relay networks," submitted to IEEE Trans. on Info. Theory, Nov. 2011, available at http://arxiv.org/abs/1111.2837v1.

[13] S. H. Lim, Y. Kim, A. El Gamal, and S. Chung, "Noisy network coding," IEEE Trans. Info. Theory, vol. 57, no. 5, pp. 3132 -3152, May 2011.

[14] A. E. Gamal and Y.-H. Kim, Network Information Theory, 1st ed. Cambridge University Press, 2011. 